\title{
Readout of Quantum Screening Effects Using a Time-Dependent Probe
}

\author{
Nastaran Dashti®, ${ }^{1}$ Matteo Acciai $\odot,{ }^{1}$ Sara Kheradsoud, ${ }^{2}$ Maciej Misiorny $\odot,{ }^{1}$ \\ Peter Samuelsson $\oplus^{2},{ }^{1}$ and Janine Splettstoesser $\oplus^{1}$ \\ ${ }^{1}$ Department of Microtechnology and Nanoscience (MC2), Chalmers University of Technology, \\ S-412 96 Göteborg, Sweden \\ ${ }^{2}$ Physics Department and NanoLund, Lund University, S-221 00 Lund, Sweden
}

(Received 9 July 2021; accepted 9 November 2021; published 10 December 2021)

\begin{abstract}
In voltage- and temperature-biased coherent conductors quantum screening effects occur if the conductor's transmission is energy dependent. Here, we show that an additional ac-driven terminal can act as a probe for a direct readout of such effects, hitherto unexplored. We find that screening of charges induced by the static biases impacts already their standard linear thermoelectric response coefficients due to nonlinear effects when accounting for the frequency of the time-dependent driving. Those effects should be observable under realistic experimental conditions and can literally be switched on and off with the ac driving.
\end{abstract}

DOI: $10.1103 /$ PhysRevLett.127.246802

In recent years, there has been a growing interest in the field of nanoscale thermoelectrics [1]: by exploiting the features of nanoscale conductors - such as their energydependent transmission properties, single-particle effects, and even quantum interference effects-novel principles for electric heat-to-work conversion are currently explored. In contrast to analogous macroscopic devices, which are typically well characterized by their linear thermoelectric properties, the nonlinear response plays an important role for these nanoscale conductors, where applied temperature or voltage differences can easily be of the order of internal energy scales. However, the nonlinear operation of these devices goes along with complex quantum screening effects in the conductor, which impact their transmission properties [2-6]. Despite their relevance, these effects, in particular those related to quantum (compared to geometrical) capacitances, have been little explored so far [7], because they are easily masked by other higher-order effects in experiments. Moreover, temperature-biasinduced screening effects have to our knowledge not been experimentally accessed at all.

In this Letter, we propose a mesoscopic setup that can be exploited to read out these quantum screening effects. It consists of a thermally and electrically biased thermoelectric two-terminal conductor, additionally ac driven by a third local, capacitively coupled terminal. The proposed device, as shown in Fig. 1, has an arbitrary

Published by the American Physical Society under the terms of the Creative Commons Attribution 4.0 International license. Further distribution of this work must maintain attribution to the author(s) and the published article's title, journal citation, and DOI. Funded by Bibsam. energy-dependent transmission, $D(E)$. One possible, simple example for such a conductor could be a quantum point contact (QPC) [8-12]. Away from equilibrium, due to an applied voltage or temperature bias, charge is accumulated at the energy-dependent scatterer, acting as a quantum capacitor. The accumulated charge is screened by charge redistributions at nearby metallic contacts and gates, coupled via geometrical capacitances to the scatterer, and by displacement currents flowing from the contacts. Treating

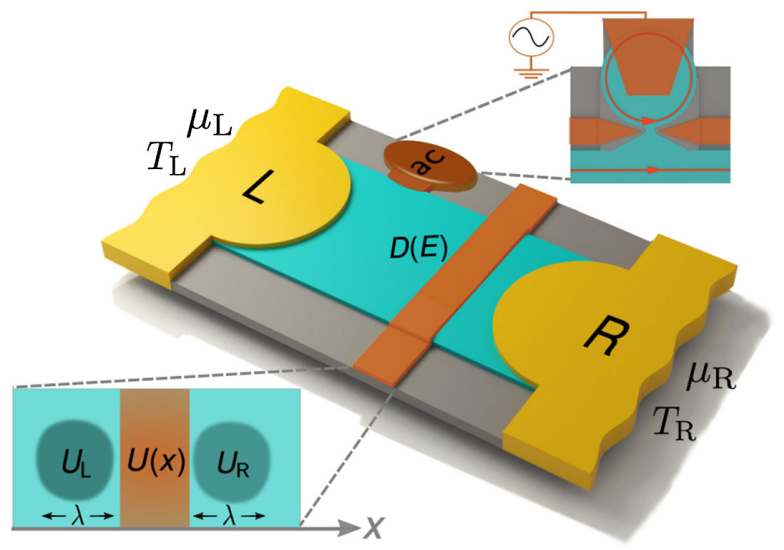

FIG. 1. Schematic of a coherent mesoscopic conductor, connected to left and right contacts with electrochemical potentials $\mu_{L}, \mu_{R}$ and temperatures $T_{L}, T_{R}$. A third, ac-driven terminal is coupled capacitively only. See the upper inset for the example of a mescocopic capacitor as the ac source. The coherent conductor has an energy-dependent transmission $D(E)$ (realized, e.g., by a QPC). Lower inset: the potential $U(x)$ creating the energydependent scattering region, as well as screening potentials $U_{L, R}$ occurring within the screening length $\lambda$ are schematically indicated. 
the electron-electron interactions at a mean-field level [13], the result of screening is a shift of the electrostatic potential in the conductor, which hence modifies its transmission properties depending on the applied electrical and thermal biases, $D(E) \equiv D(E,\{V, \Delta T\})[2,6,14,15]$.

We find that screening effects due to the stationary biases can already be made visible as corrections to the standard linear thermoelectric response of the two-terminal conductor to voltage and temperature biases. These surprising corrections stem from the time-dependent driving, which is locally applied via a third terminal and which could, e.g., be realized by a mesoscopic capacitor $[7,16,17]$ in the quantum Hall regime [8,9]. More specifically, the discovered corrections to the thermoelectric linear-response coefficients are directly proportional to different quantum screening coefficients, which usually only play a role in the nonlinear thermoelectric response of stationary conductors $[2,6,13-15,18-20]$. In the latter case they occur as higherorder correction effects in the static biases, which are hard to extract from an experiment. In contrast, the correction terms identified here are nonlinear only when accounting for the ac-driving frequency as one of the affinities in a generalized thermoelectric framework [21]. Screening corrections can hence be switched on and off by adding a local ac driving and they can thus directly be extracted by comparing standardly detected linear-response coefficients, in the presence and in the absence of the driving.

In the following, we derive charge and heat currents flowing in the time-dependently driven setup shown in Fig. 1, using a Floquet scattering matrix approach [22-25] and carefully considering geometrical and quantum screening effects induced by both voltage and temperature biases. We then elaborate on concrete strategies to exploit the interplay between screening effects and ac driving in order to read out until now elusive screening coefficients.

Charge and heat currents in the driven conductor.-We consider a coherent mesoscopic conductor connecting two electronic contacts, $L$ and $R$, via a scattering region with energy-dependent transmission $D(E)$. Here, we assume contact $L$ to be electrically grounded, i.e., $\mu_{L}=\mu_{0}$, and kept at temperature $T_{L}=T_{0}$, while electrochemical potential and temperature in contact $R$ are assumed to be $\mu_{R}=\mu_{0}+e V$ and $T_{R}=T_{0}-\Delta T$, respectively. Here, $-e$ is the charge of the electron, with $e>0$. In what follows, we set $\mu_{0} \equiv 0$ as the reference energy. Furthermore, the conductor is subjected to a controlled, local ac driving applied via a third, capacitively coupled contact.

We choose contact $R$ to be the one where the timeaveraged charge and energy currents are detected, $I \equiv I_{R}$ and $I^{E} \equiv I_{R}^{E}$. For the conductor shown in Fig. 1, they read [24-26]

$$
I=I_{\mathrm{ac}}+\frac{e}{h} \int d E D(E)\left\{f_{R}(E)-f_{L}(E)\right\},
$$

$$
I^{E}=I_{\mathrm{ac}}^{E}-\frac{1}{h} \int d E E D(E)\left\{f_{R}(E)-f_{L}(E)\right\},
$$

with $f_{\alpha}(E)=\left[1+\exp \left(\left[E-\mu_{\alpha}\right] / k_{B} T_{\alpha}\right)\right]^{-1}$. Here, we have split the full currents into a contribution arising from the applied stationary temperature and voltage biases [second part of the right-hand sides of Eqs. (1)] and contributions, $I_{\mathrm{ac}}$ and $I_{\mathrm{ac}}^{E}$, arising from a time average of the ac-driving induced currents. The latter are given by

$$
\begin{aligned}
& I_{\mathrm{ac}}=\frac{e}{h} \sum_{n=-\infty}^{\infty} \int d E\left|S_{n}(E)\right|^{2} D(E) \Delta f_{0}\left(E_{n}\right), \\
& I_{\mathrm{ac}}^{E}=-\frac{1}{h} \sum_{n=-\infty}^{\infty} \int d E\left|S_{n}(E)\right|^{2} D(E) E \Delta f_{0}\left(E_{n}\right) .
\end{aligned}
$$

Here, $S_{n}(E)$ is the $n$th Fourier component of the scattering matrix of the driven region, see Refs. [27,28] for explicit examples. The function $\Delta f_{0}\left(E_{n}\right)=f_{0}(E)-$ $f_{0}\left(E_{n}\right)$ is a difference between equilibrium Fermi functions, $f_{L}(E) \equiv f_{0}(E)=\left[1+\exp \left(E / k_{B} T_{0}\right)\right]^{-1}$ at energies $E$ and $E_{n}=E+n \hbar \Omega$, differing by an integer multiple of the ac-driving frequency $\Omega$. Equation (2) relies on the assumption of no backscattering from the conductor towards the source, which is met, e.g., in chiral systems. In order to obtain the heat current from the expressions given in Eq. (1), one needs to evaluate $J=I^{E}-V I$, and analogous expressions for the separate components of the heat current arising from the stationary biases or ac-driving, alone.

Linear thermoelectric response to $V$ and $\Delta T$.- Starting from the general results for charge and energy currents, Eq. (1), we derive expressions for $I$ and $J$ to leading order in the applied biases, $V$ and $\Delta T$, but without expanding in the driving frequency

$$
\left(\begin{array}{c}
I \\
J
\end{array}\right)=\left(\begin{array}{c}
I_{\mathrm{ac}}^{\mathrm{dir}} \\
I_{\mathrm{ac}}^{E, \mathrm{dir}}
\end{array}\right)+\left(\begin{array}{cc}
G+G_{\mathrm{ac}} & L+L_{\mathrm{ac}} \\
M+M_{\mathrm{ac}} & K+K_{\mathrm{ac}}
\end{array}\right)\left(\begin{array}{c}
V \\
\Delta T
\end{array}\right) .
$$

To obtain this equation, we expand the Fermi functions to linear order in the biases, as well as the energy-dependent transmission probability, which depends on the biases due to screening [2,6,13-15,18-20,29]:

$D(E,\{V, \Delta T\})=D_{0}(E)+\frac{1}{2} \frac{d D_{0}}{d E}\left(\xi e V+\chi k_{B} \Delta T\right)$.

Here, we introduce $D_{0}(E)=D(E,\{0,0\})$. The coefficient $0 \leq \xi \leq 1$ is bounded from above by gauge invariance, while $\chi$ can have any sign and is not bounded. We evaluate the screening coefficients due to voltage and temperature biases, $\chi$ and $\xi$, for the explicit example of a QPC in the last part of this Letter. In principle, screening at the QPC of the electrons and holes injected from the local ac driving 
should also be accounted for. However, we focus on driving sources operated such that this dynamical, ac-screening effect is negligible [29].

The first terms appearing in Eq. (3) are to leading order not affected by the applied biases, that is $I_{\mathrm{ac}}^{\mathrm{dir}}=\left.I_{\mathrm{ac}}\right|_{\Delta T, V=0}$ and $I_{\mathrm{ac}}^{E \text {,dir }}=\left.I_{\mathrm{ac}}^{E}\right|_{\Delta T, V=0}$, with $I_{\mathrm{ac}}, I_{\mathrm{ac}}^{E}$ given in Eqs. (2a) and (2b). Furthermore, the matrix elements $G, L, M$, and $K$ are the standard, linear response, thermoelectric coefficients

$G=\frac{e^{2}}{h} \mathcal{I}_{0}, \quad L=\frac{M}{T_{0}}=-\frac{e}{h} k_{B} \mathcal{I}_{1}, \quad K=\frac{1}{h}\left(k_{B}^{2} T_{0}\right) \mathcal{I}_{2}$,

(see, e.g., Ref. [1] for a review) with

$$
\mathcal{I}_{\ell}=\int_{-\infty}^{\infty} d E D_{0}(E)\left(\frac{E}{k_{B} T_{0}}\right)^{\ell}\left(-\frac{\partial f_{0}(E)}{\partial E}\right) .
$$

Here, $G$ is the electrical conductance, $K$ the thermal one, and $L, M$ the thermoelectric coefficients related to the Seebeck and Peltier coefficients. We emphasize that none of these quantities is affected by screening effects. Of main interest here, are the coefficients $G_{\mathrm{ac}}, L_{\mathrm{ac}}, M_{\mathrm{ac}}$, and $K_{\mathrm{ac}}$, which modify the standard linear response result and which may, in general, depend nonlinearly on the ac-driving frequency. These coefficients all arise from the interplay between the nonequilibrium induced screening effects and the time-dependent driving. Namely, the applied biases lead to a voltage- and temperature-dependent transmission probability, $D(E)$, which in turn modifies the currents injected due to the local time-dependent driving, when they are scattered at the conductor. The expressions for the coefficients are

$$
\begin{aligned}
G_{\mathrm{ac}} & =\xi \frac{e^{2}}{2 h} \mathcal{J}_{0}, \quad L_{\mathrm{ac}}=\chi \frac{k_{B} e}{2 h} \mathcal{J}_{0}, \\
M_{\mathrm{ac}} & =-\xi \frac{k_{B} e}{2 h} T_{0} \mathcal{J}_{1}, \quad K_{\mathrm{ac}}=-\chi \frac{k_{B}^{2} T_{0}}{2 h} \mathcal{J}_{1},
\end{aligned}
$$

where

$$
\mathcal{J}_{\ell}=\sum_{n} \int d E\left|S_{n}(E)\right|^{2} \frac{d D_{0}(E)}{d E}\left(\frac{E}{k_{B} T_{0}}\right)^{\ell} \Delta f_{0}\left(E_{n}\right) .
$$

Interestingly, from Eq. (7) we see that the charge-current and heat-current coefficients are related in a simple way:

$$
\frac{G_{\mathrm{ac}}}{e \xi}=\frac{L_{\mathrm{ac}}}{k_{B} \chi}, \quad \frac{M_{\mathrm{ac}}}{e \xi}=\frac{K_{\mathrm{ac}}}{k_{B} \chi} .
$$

This derives from the fact that the two pairs of coefficients, $G_{\mathrm{ac}}, M_{\mathrm{ac}}$ and $L_{\mathrm{ac}}, K_{\mathrm{ac}}$, respectively, stem from voltage- and temperature-induced screening effects.
Equation (9), demonstrates that the total coefficient matrix in Eq. (3) does not satisfy Onsager's symmetry relations. We stress that this breakdown is to be expected, due to the external driving breaking time-reversal symmetry. Onsager symmetries can be recovered by treating the frequency as an affinity in the adiabatic regime [21].

Weak thermoelectric effect.-The origin of the coefficients in Eq. (7) as an interplay between the screening effects and the ac-induced currents becomes formally explicit in the limit of a weak thermoelectric effect, that is, for a conductor with a smooth energy dependence. In this limit, we can expand the transmission probability to first order in energy as $D_{0}(E) \approx D_{0}+E D_{0}^{\prime}$, where $D_{0} \equiv D_{0}(0)$ and $D_{0}^{\prime} \equiv d D_{0}(E) /\left.d E\right|_{E=0}$. Inserting this expansion into the coefficients in Eq. (7) we arrive at

$$
G_{\mathrm{ac}}=-\frac{h}{2 e^{2}} \frac{L \xi}{\mathcal{L}_{0} T_{0}} I_{\mathrm{ac}, 0}^{\mathrm{dir}}, \quad M_{\mathrm{ac}}=-\frac{h}{2 e^{2}} \frac{L \xi}{\mathcal{L}_{0} T_{0}} J_{\mathrm{ac}, 0}^{\mathrm{dir}},
$$

and equivalent relations for $L_{\mathrm{ac}}$ and $K_{\mathrm{ac}}$ from Eq. (9). Here, the thermoelectric coefficient is given by $L=$ $-\left(e \pi^{2} / 3 h\right) k_{B}^{2} T_{0} D_{0}^{\prime}$ in accordance with Mott's law and the Lorenz number is defined as $\mathcal{L}_{0}=\left(\pi^{2} k_{B}^{2} / 3 e^{2}\right)$. Importantly, the corrections to all response coefficients, $G_{\mathrm{ac}}, L_{\mathrm{ac}}, M_{\mathrm{ac}}$, and $K_{\mathrm{ac}}$ become particularly simple in this regime. They are proportional to the screening coefficients $\xi$ and $\chi$, and to the same unperturbed thermoelectric coefficient $L$. Furthermore, they are proportional to the bare charge or heat currents from the time-dependent driving, $I_{\mathrm{ac}, 0}^{\mathrm{dir}}$ or $J_{\mathrm{ac}, 0}^{\mathrm{dir}}$, that would flow into contact $R$ for a completely open conductor, $D(E) \rightarrow 1$, and in the absence of stationary biases. Note, however, that $I_{\mathrm{ac}, 0}^{\mathrm{dir}} \equiv 0$, due to the fact that the additional time-dependent driving is local and purely ac. This means that in order to obtain nonvanishing corrections to the linear thermoelectric response coefficients of the charge current, $G_{\mathrm{ac}}$ and $L_{\mathrm{ac}}$, the energy dependence of the conductor's transmission probability needs to be at least quadratic.

Sensing of quantum screening effects.-In typical, purely statically biased conductors, the screening effects introduced above occur as higher-order corrections in $\Delta T$ and $V$ $[2,6,14,15]$, which are hard to clearly identify. Indeed, screening effects due to a temperature bias have not been observed so far. In the present Letter, we propose to exploit the interplay between local ac-driving and quantum screening effects to read out the latter from the modifications of the linear-response coefficients, $G_{\mathrm{ac}}, L_{\mathrm{ac}}, M_{\mathrm{ac}}$, and $K_{\mathrm{ac}}$. Note that these are not simply uncontrolled small corrections to the standard linear-response coefficients, but can be switched on and off at will with the ac driving. For the specific readout, we distinguish two situations: (i) the weak thermoelectric case, where at the same time the driving properties are well known, and (ii) the general case of arbitrary $D(E)$, where we do not assume a detailed knowledge of the driving features either. 
Case (i) requires the possibility of detecting the heat current response of the conductor. Equations (9) and (10) determine the modifications of the linear-response coefficients. While $I_{\mathrm{ac}, 0}^{\mathrm{dir}} \equiv 0$, one can determine $J_{\mathrm{ac}, 0}^{\mathrm{dir}}$ from a heat current measurement where the ac driving is applied, but no stationary biases. A stationary charge-current measurement in the presence of a temperature bias yields $L$. With this, one can subsequently directly extract the coefficients $\xi$ and $\chi$ from a detection of $M+M_{\mathrm{ac}}$ and $K+$ $K_{\text {ac }}$ compared to $M$ and $K$ in the absence of an ac driving.

In case (ii), the functions $\mathcal{J}_{0}$ and $\mathcal{J}_{1}$ are not necessarily known. An experiment could then have two strategies to proceed: either a measurement of all four coefficients, $G_{\mathrm{ac}}$, $L_{\mathrm{ac}}, M_{\mathrm{ac}}$, and $K_{\mathrm{ac}}$, gives access to the four unknown functions $\chi, \xi, \mathcal{J}_{0}$, and $\mathcal{J}_{1}$, allowing one to determine $\chi$ and $\xi$, separately. Otherwise, in an experiment, e.g., restricted to a measurement of charge-current coefficients only, one could extract the ratio

$$
\frac{\chi}{\xi}=\frac{e}{k_{B}} \frac{L_{\mathrm{ac}}}{G_{\mathrm{ac}}}
$$

This would give access to, until now undetected, quantum screening properties due to a thermal bias, as it will be shown in the following example of a QPC.

Quantum point contact.-As an explicit example, we here consider a scattering region created by a gate-tunable QPC. It can be described by an inverted parabola potential $U(x)=\epsilon-m \omega^{2} x^{2} / 2$, where $m$ is the effective electron mass, $\omega$ determines the smoothness of the barrier as $\gamma=2 \hbar \omega$, and $\epsilon$ is a threshold energy. The QPC's equilibrium transmission probability is then given by [9]

$$
D_{0}(E)=\frac{1}{1+\exp [-(E-\epsilon) / \gamma]} .
$$

In order to evaluate screening effects, we follow Refs. [14,15] and consider a model of the QPC with two constant potential regions, where the charge is not perfectly screened, one on each side of the QPC, see the inset of Fig. 1. Their size is given by the screening length $\lambda$. We consider a spatially symmetric setup, where the constant-potential regions are equally capacitively coupled to both the QPC split-gate electrodes, with capacitance $C_{g}$, and to the respective electronic contact, with capacitance $C$. All other capacitive couplings are assumed to have a negligibly small influence on the screening properties. The detailed derivation of the QPC's scattering properties within a semi-classical, WKB approach is shown in the Supplemental Material $[29,30]$. It yields explicit expressions for the dimensionless coefficients $\chi$ and $\xi$, introduced in Eq. (4), given by

$$
\xi=\frac{2 C+\mathcal{D}}{2 C+\mathcal{D}+2 C_{g}}, \quad \chi=\frac{\mathcal{D}^{E}}{2 C+\mathcal{D}+2 C_{g}} .
$$

They contain both the geometric capacitances $C$ and $C_{g}$, which can be obtained via a careful modeling of the geometry of the actual experimental device (see, e.g., Ref. [31]), as well as $\mathcal{D}=-e^{2} \int d E \nu(E) \partial f_{0} / \partial E$ and $\mathcal{D}^{E}=-e^{2} \int d E\left[E /\left(k_{B} T_{0}\right)\right] \nu(E) \partial f_{0} / \partial E$, which are due to quantum screening. In particular, $\mathcal{D}$ is the so-called quantum capacitance [7,16,32,33], while $\mathcal{D}^{E}$ (also having units of a capacitance) is related to the charge pile-up in the system due to temperature variations $[6,15]$. They are both quantum properties, as they involve $\nu(E)$, that is the total density of states in the two constant-potential regions, $U_{L, R}$. The density of states is given by $[14,34,35]$

$\nu(E)=\frac{1}{\gamma \pi} \times \begin{cases}\operatorname{arcosh}\left[\sqrt{\frac{E_{\lambda}}{\epsilon-E}}\right], & \text { for } \epsilon-E_{\lambda}<E<\epsilon \\ \operatorname{arsinh}\left[\sqrt{\frac{E_{\lambda}}{E-\epsilon}}\right], & \text { for } E>\epsilon .\end{cases}$

Here, $E_{\lambda} \equiv m \omega^{2} \lambda^{2} / 2=\gamma^{2}\left[m \lambda^{2} /\left(8 \hbar^{2}\right)\right] \equiv \gamma^{2} / E_{\mathrm{box}}$, where $E_{\text {box }}$ and $E_{\lambda}$ are two energy scales related to the screening length $\lambda$, indicated in the lower inset of Fig. 1. Importantly, the expression in Eq. (13) clearly shows that the factor $\chi / \xi=\mathcal{D}^{E} /(2 C+\mathcal{D})$, that is most easily accessible by the above described readout scheme (ii), gives access to quantum screening properties due to a thermal bias, encoded in $\mathcal{D}^{E}$.

Conveniently, $\xi$ and $\chi$ can be expressed [29] in terms of three dimensionless energy parameters $\epsilon /\left(k_{B} T_{0}\right)$, $E_{\text {box }} /\left(k_{B} T_{0}\right)$, and $\gamma /\left(k_{B} T_{0}\right)$, as well as in terms of two dimensionless, capacitive parameters $C_{g} / C$, and $C_{\mathcal{D}} / C$, where $C_{\mathcal{D}}=e^{2} /\left(8 \pi k_{B} T_{0}\right)$. The combination $C_{\mathcal{D}} \gamma / E_{\mathrm{box}}$, containing the screening length $\lambda$, gives the typical magnitude of the quantum capacitances $\mathcal{D}$ and $\mathcal{D}^{E}$ [29]. From Eq. (13) it follows that in the limit of dominant capacitive coupling to the gate, $C_{g} \gg C, \mathcal{D}, \mathcal{D}^{E}$ both coefficients are small, $\xi, \chi \ll 1$, leading to a tiny modification of the transmission with respect to $D_{0}(E)$. In the regime $C \gg C_{g}, \mathcal{D}, \mathcal{D}^{E}$, with dominant capacitive coupling to the contacts, $\xi \rightarrow 1$ and the internal potentials $U_{L, R}$ are shifted by the same amount as the electrochemical potentials $\mu_{L, R}$. However $\chi \ll 1$, i.e., the effect of temperature is small. For the regime of dominant quantum capacitances, $\mathcal{D}, \mathcal{D}^{E} \gg C, C_{g}$, both coefficients $\chi, \xi$ can be of order one. Taken together, the effect on the transmission due to applied bias or temperature is determined by the relation between quantum and geometric capacitances. Note that both the magnitude of the quantum capacitance, resulting from imperfect screening at the QPC, as well as the classical, capacitive couplings between different parts of the conductor are affected by the strength of electron-electron interactions.

In Fig. 2, we plot both $\xi$ and $\chi$ as a function of $\epsilon$ for a representative set of parameters. The dependence on $\epsilon$ in 

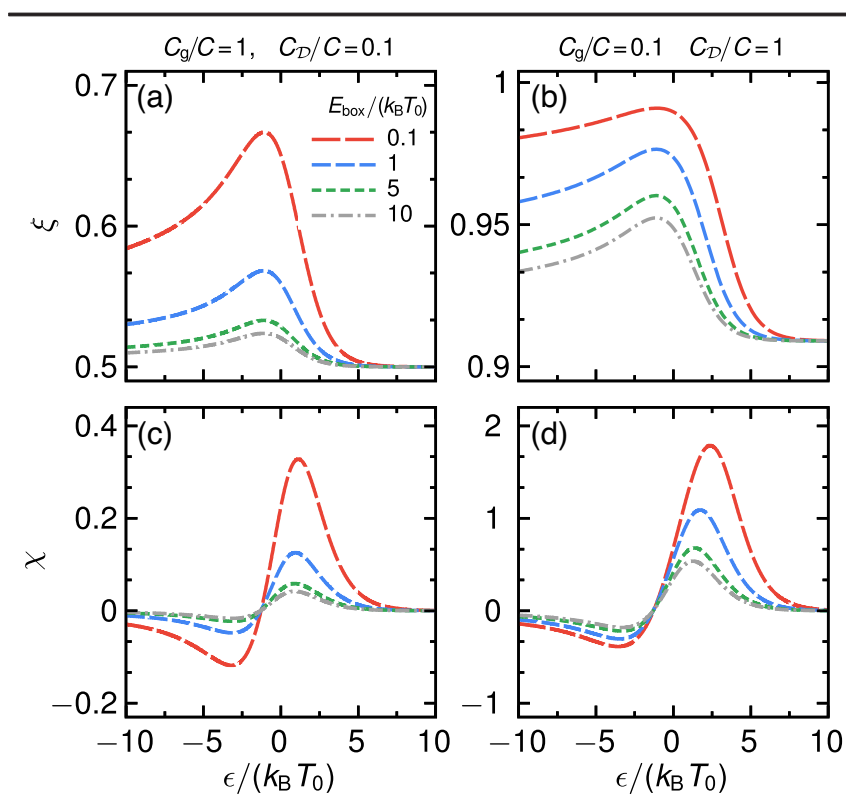

FIG. 2. Coefficients $\xi$, in (a),(b), and $\chi$, in (c),(d) as a function of $\epsilon /\left(k_{B} T_{0}\right)$ for a set of different $E_{\mathrm{box}} /\left(k_{B} T_{0}\right)$, see legend in (a), and for different values of the parameters $C_{g} / C$ and $C_{\mathcal{D}} / C$. Here, the smoothness of the barrier is $\gamma /\left(k_{B} T_{0}\right)=0.1$.

these plots is entirely due to quantum capacitances, which, unlike geometric ones, depend on the transmission properties of the conductor. We see that $\xi$ shows a qualitatively similar behavior in both panels, Figs. 2(a) and 2(b), with a maximum around $\epsilon=0$, approaching $C /\left(C+C_{g}\right)$ for $\epsilon /\left(k_{B} T_{0}\right) \rightarrow \infty$ and decaying slowly with increasingly negative $\epsilon$. The magnitude of the variations with $\epsilon$ is however larger for $C_{g} \gg C_{\mathcal{D}}$. The trend is opposite for $\chi$, in Figs. 2(c) and 2(d), where larger variations with $\epsilon$ occur for $C_{\mathcal{D}} \gg C_{g}$. Overall, $\chi$ shows a qualitatively similar, alternating-sign behavior in both panels, with a negative peak at $\epsilon<0$ and a positive peak at $\epsilon>0$, both of the order of $k_{B} T_{0}$ away from the origin. For $\epsilon /\left(k_{B} T_{0}\right) \rightarrow \pm \infty$, $\chi$ approaches zero.

Conclusions.-We have shown how the interplay between a local ac driving and quantum screening effects due to stationary thermal and electrical biases impacts the standard, stationary linear response of a thermoelectric conductor. We use this to put forward a proposal for the direct readout of-until now elusive-quantum screening effects, from tunable modifications of linear-response coefficients. We expect presently available experimental techniques [36-40] to allow for the proposed readout of quantum screening effects. For the same parameters as in Fig. 2 and considering as a probe a mesoscopic capacitor with driving frequency around $1 \mathrm{GHz}$ and escape time of $30 \mathrm{ps}$ [41] and a static voltage bias of $10 \mu \mathrm{V}$, we get a rough estimate of $5 \mathrm{pA}$ and $1 \mathrm{fW}$ for the corrections to the charge and heat currents, respectively. These values are increased by reducing $\gamma$ (i.e., sharpening the energy dependence of the QPC transmission). The findings of such an experiment could test predictions for screening coefficients, as they are shown in Fig. 2.

We furthermore foresee that with this very same setup, known screening effects can be used to perform tomography on single-electron sources (attached to the third contact in our scheme) [39]. Also, the controlled modification of thermoelectric response coefficients by the driving is expected to be of interest for the improvement of heat engines [42].

We thank F. Taddei, R. S. Whitney, G. Haack, and S. Lara-Avila for interesting discussions and G. Fève and D. Sánchez for useful comments on the manuscript. Funding from the Knut and Alice Wallenberg Foundation through the Academy Fellow program (J.S., N.D., M. A., and M. M.), from the European Union's H2020 research and innovation program under Grant Agreement No. 862683 UltraFastNano (M. A. and J. S.), and from the Swedish VR are gratefully acknowledged.

[1] G. Benenti, G. Casati, K. Saito, and R. S. Whitney, Fundamental aspects of steady-state conversion of heat to work at the nanoscale, Phys. Rep. 694, 1 (2017).

[2] T. Christen and M. Büttiker, Gauge-invariant nonlinear electric transport in mesoscopic conductors, Europhys. Lett. 35, 523 (1996).

[3] M. Büttiker and D. Sánchez, Interaction-induced magnetic field asymmetry of nonlinear mesoscopic electrical transport, Int. J. Quantum Chem. 105, 906 (2005).

[4] M. Büttiker and T. Christen, Admittance and nonlinear transport in quantum wires, point contacts, and resonant tunneling barriers, in Mesoscopic Electron Transport, edited by L. L. Sohn, L. P. Kouwenhoven, and G. Schön (Springer Netherlands, Dordrecht, 1997), pp. 259-289.

[5] D. Sánchez and M. Büttiker, Magnetic-Field Asymmetry of Nonlinear Mesoscopic Transport, Phys. Rev. Lett. 93, 106802 (2004).

[6] D. Sánchez and R. López, Scattering Theory of Nonlinear Thermoelectric Transport, Phys. Rev. Lett. 110, 026804 (2013).

[7] J. Gabelli, G. Fève, J.-M. Berroir, B. Plaçais, A. Cavanna, B. Etienne, Y. Jin, and D. C. Glattli, Violation of Kirchhoff's laws for a coherent $R C$ circuit, Science 313, 499 (2006).

[8] H. A. Fertig and B. I. Halperin, Transmission coefficient of an electron through a saddle-point potential in a magnetic field, Phys. Rev. B 36, 7969 (1987).

[9] M. Büttiker, Quantized transmission of a saddle-point constriction, Phys. Rev. B 41, 7906 (1990).

[10] H. van Houten, L. W. Molenkamp, C. W. J. Beenakker, and C. T. Foxon, Thermo-electric properties of quantum point contacts, Semicond. Sci. Technol. 7, B215 (1992).

[11] R. S. Whitney, Most Efficient Quantum Thermoelectric at Finite Power Output, Phys. Rev. Lett. 112, 130601 (2014).

[12] R.S. Whitney, Finding the quantum thermoelectric with maximal efficiency and minimal entropy production at given power output, Phys. Rev. B 91, 115425 (2015). 
[13] M. Büttiker, Capacitance, admittance, and rectification properties of small conductors, J. Phys. Condens. Matter 5, 9361 (1993).

[14] M. H. Pedersen, S. A. van Langen, and M. Büttiker, Charge fluctuations in quantum point contacts and chaotic cavities in the presence of transport, Phys. Rev. B 57, 1838 (1998).

[15] J. Meair and P. Jacquod, Scattering theory of nonlinear thermoelectricity in quantum coherent conductors, J. Phys. Condens. Matter 25, 082201 (2013).

[16] M. Büttiker, H. Thomas, and A. Prêtre, Mesoscopic capacitors, Phys. Lett. A 180, 364 (1993).

[17] G. Fève, A. Mahé, J.-M. Berroir, T. Kontos, B. Plaçais, D. C. Glattli, A. Cavanna, B. Etienne, and Y. Jin, An ondemand coherent single-electron source, Science 316, 1169 (2007).

[18] M. Büttiker, A. Prêtre, and H. Thomas, Dynamic Conductance and the Scattering Matrix of Small Conductors, Phys. Rev. Lett. 70, 4114 (1993).

[19] T. Christen and M. Büttiker, Low Frequency Admittance of a Quantum Point Contact, Phys. Rev. Lett. 77, 143 (1996).

[20] C. Texier and J. Mitscherling, Nonlinear conductance in weakly disordered mesoscopic wires: Interaction and magnetic field asymmetry, Phys. Rev. B 97, 075306 (2018).

[21] M. F. Ludovico, F. Battista, F. von Oppen, and L. Arrachea, Adiabatic response and quantum thermoelectrics for acdriven quantum systems, Phys. Rev. B 93, 075136 (2016).

[22] M. Büttiker, Four-Terminal Phase-Coherent Conductance, Phys. Rev. Lett. 57, 1761 (1986).

[23] P. N. Butcher, Thermal and electrical transport formalism for electronic microstructures with many terminals, J. Phys. Condens. Matter 2, 4869 (1990).

[24] Ya. M. Blanter and M. Büttiker, Shot noise in mesoscopic conductors, Phys. Rep. 336, 1 (2000).

[25] M. V. Moskalets, Scattering Matrix Approach to NonStationary Quantum Transport (World Scientific Publishing Company, Singapore, 2011).

[26] Y. V. Nazarov and Y.M. Blanter, Quantum Transport: Introduction to Nanoscience (Cambridge University Press, Cambridge, England, 2009).

[27] M. Misiorny, G. Fève, and J. Splettstoesser, Shaping charge excitations in chiral edge states with a time-dependent gate voltage, Phys. Rev. B 97, 075426 (2018).

[28] N. Dashti, M. Misiorny, S. Kheradsoud, P. Samuelsson, and J. Splettstoesser, Minimal excitation single-particle emitters: Comparison of charge-transport and energy-transport properties, Phys. Rev. B 100, 035405 (2019).
[29] See Supplemental Material at http://link.aps.org/ supplemental/10.1103/PhysRevLett.127.246802 for further details.

[30] J. N. L. Connor, On the analytical description of resonance tunnelling reactions, Mol. Phys. 15, 37 (1968).

[31] C. Déprez, L. Veyrat, H. Vignaud, G. Nayak, K. Watanabe, T. Taniguchi, F. Gay, H. Sellier, and B. Sacépé, A tunable Fabry-Pérot quantum Hall interferometer in graphene, Nat. Nanotechnol. 16, 555 (2021).

[32] S. Luryi, Quantum capacitance devices, Appl. Phys. Lett. 52, 501 (1988).

[33] A. Prêtre, H. Thomas, and M. Büttiker, Dynamic admittance of mesoscopic conductors: Discrete-potential model, Phys. Rev. B 54, 8130 (1996).

[34] E. P. Wigner, Lower limit for the energy derivative of the scattering phase shift, Phys. Rev. 98, 145 (1955).

[35] F. T. Smith, Lifetime matrix in collision theory, Phys. Rev. 118, 349 (1960).

[36] C. Bäuerle, D. C. Glattli, T. Meunier, F. Portier, P. Roche, P. Roulleau, S. Takada, and X. Waintal, Coherent control of single electrons: A review of current progress, Rep. Prog. Phys. 81, 056503 (2018).

[37] B. Brun, F. Martins, S. Faniel, A. Cavanna, C. Ulysse, A. Ouerghi, U. Gennser, D. Mailly, P. Simon, S. Huant, M. Sanquer, H. Sellier, V. Bayot, and B. Hackens, Thermoelectric Scanning-Gate Interferometry on a Quantum Point Contact, Phys. Rev. Applied 11, 034069 (2019).

[38] J. Dubois, Vers une source mésoscopique à n-électrons basée sur des pulses de tensions Lorentziens, Ph.D. thesis, Université Pierre et Marie Curie - Paris VI, 2012.

[39] J. D. Fletcher, P. See, H. Howe, M. Pepper, S. P. Giblin, J. P. Griffiths, G. A. C. Jones, I. Farrer, D. A. Ritchie, T. J. B. M. Janssen, and M. Kataoka, Clock-Controlled Emission of Single-Electron Wave Packets in a Solid-State Circuit, Phys. Rev. Lett. 111, 216807 (2013).

[40] N. Johnson, J. D. Fletcher, D. A. Humphreys, P. See, J. P. Griffiths, G. A. C. Jones, I. Farrer, D. A. Ritchie, M. Pepper, T. J. B. M. Janssen, and M. Kataoka, Ultrafast voltage sampling using single-electron wavepackets, Appl. Phys. Lett. 110, 102105 (2017).

[41] A. Mahé, F. D. Parmentier, E. Bocquillon, J.-M. Berroir, D. C. Glattli, T. Kontos, B. Plaçais, G. Fève, A. Cavanna, and Y. Jin, Current correlations of an on-demand singleelectron emitter, Phys. Rev. B 82, 201309(R) (2010).

[42] H. Zhou, J. Thingna, P. Hänggi, J.-S. Wang, and B. Li, Boosting thermoelectric efficiency using time-dependent control, Sci. Rep. 5, 1 (2015). 